\title{
THE IMPACT OF INTEREST RATE CHANGES ON STOCK MARKET
}

\author{
Manjunatha \\ Research Scholar, \\ Dr. KUSHALAPPA. S \\ AIET, Mijar, Moodbidri. 574225 \\ Senior Assistant Professor, \\ AIET, Mijar, Moodbidri. 574225
}

\begin{abstract}
:
The goal of this study is to examine the relationships between interest rate and stock market index in India. The performance of the stock market can reflect the overall performance of a country's economy. Similarly the stock index in a particular sector can reflect the performance of the particular sector, for example, the plantation index. When palm oil-related companies are making huge profits, plantation index will tend to go up. When the stock market is doing well, it may imply that the economy is experiencing high growth. There are many factors that affect the performance of the stock market; internal factors and external factors to company. The risk investment may arise from both the factors. Risk arises due to internal factors can be controlled by an efficient portfolio management but the risk arises due to external factors cannot be controlled by the investors. The current study deals with the impact of external factors on the performance of stock market. The external factor taken in this study is the interest rate changes. Ups and downs in the interest rates will have an impact on the performance of the stocks in the stock market. A particular stock may perform better due to increase in the interest rate or it may perform poor after the interest rates have been increased by the RBI. Here an attempt is made study the behavior of stock market for changes in the interest rates.
\end{abstract}

KEY WORDS: Interest rates, stock market, stock returns

\section{INTRODUCTION:}

The goal of this study is to examine the relationships between interest rate and stock market index in India. The performance of the stock market can reflect the overall performance of a country's economy. Similarly the stock index in a particular sector can reflect the performance of the particular sector, for example, the plantation index. When palm oil-related companies are making huge profits, plantation index will tend to go up. When the stock market is doing well, it may imply that the economy is experiencing high growth. There are many factors that affect the performance of the stock market; internal factors and external factors to company. The risk investment may arise from both the factors. Risk arises due to internal factors can be controlled by an efficient portfolio management but the risk arises due to external factors cannot be controlled by the investors. The current study deals with the impact of external factors on the performance of stock market. The external factor taken in this study is the interest rate changes. Ups and downs in the interest rates will have an impact on the performance of the stocks in the stock market. A particular stock may perform better due to increase in the interest rate or it may perform poor after the interest rates have been increased by the RBI. Here an attempt is made study the behavior of stock market for changes in the interest rates. 


\section{OBJECTIVE OF THE STUDY:}

- To study the relationship between stock market returns with respect to changes in interest rates.

- To analyse the impact of increase and decrease in the interest rates on stock market

- To study whether the changes in interest rate considered to be beneficial or loss to the investors.

- To offer meaningful suggestions to investors based on the study

\section{METHODOLOGY:}

The entire study is based on secondary data, extracted from websites like Bombay Stock Exchange (BSE), Reserve Bank of India (RBI), books and journals. The sample size is 30 BSE listed companies. The study covers interest rate changes; decrease in the interest rates and increase in the interest rate...The statistical tools like, average return and standard deviation, are being used for the purpose of making the analysis more effective.

\section{TOOLS USED FOR ANALYSIS:}

Return of the stock is being calculated based on the daily share prices by using the following formula.

$$
\begin{aligned}
& \text { Return }= \\
& \frac{\text { ClosingPrice-OpeningPrice }}{\text { OpeningPrice }} * 100
\end{aligned}
$$

Average return of the stock is been calculated based on the daily returns of the share price using the following formula.

Average $=$

$\frac{\sum_{\mathrm{i}=1}^{\mathrm{n}} \mathrm{x}}{\mathrm{n}}$

Standard deviation of the return is been calculated on the basis of daily returns of the stock price using the following formula.

$$
\sqrt{\frac{\sum(\mathrm{x}-\overline{\mathrm{x}}) 2}{\mathrm{n}}}
$$

\section{DATA ANALYSIS}

AND

\section{INTERPRETATION:}

An attempt is made here to present the analysis of data.

Table 1: Average returns and standard deviation of returns, before decrease in the interest rate

\begin{tabular}{|l|l|l|l|l|l|l|l|l|l|l|l|l|l|}
\hline $\begin{array}{l}\text { SL } \\
\text { NO }\end{array}$ & COMPANY & $\begin{array}{l}\text { Nov } \\
\mathbf{0 7}\end{array}$ & $\begin{array}{l}\text { Nov- } \\
\mathbf{0 8}\end{array}$ & $\begin{array}{l}\text { Nov- } \\
\mathbf{1 1}\end{array}$ & $\begin{array}{l}\text { Nov- } \\
\mathbf{1 2}\end{array}$ & $\begin{array}{l}\text { Nov- } \\
\mathbf{1 3}\end{array}$ & $\begin{array}{l}\text { Nov- } \\
\mathbf{1 4}\end{array}$ & $\begin{array}{l}\text { Nov- } \\
\mathbf{1 8}\end{array}$ & $\begin{array}{l}\text { Nov- } \\
\mathbf{1 9}\end{array}$ & $\begin{array}{l}\text { Nov- } \\
\mathbf{2 0}\end{array}$ & $\begin{array}{l}\text { Nov- } \\
\mathbf{2 1}\end{array}$ & AVG & STD \\
\hline 1 & Axis Bank & -3.35 & -4.52 & -3.66 & -2.65 & -1.86 & 6.63 & 3.70 & -0.12 & -0.49 & -4.05 & $\mathbf{- 0 . 7 5}$ & $\mathbf{2 . 9 7}$ \\
\hline 2 & Bajaj Auto & 1.13 & -1.63 & -1.43 & -0.67 & -0.07 & 0.49 & -0.16 & -0.71 & -1.97 & -2.04 & $\mathbf{- 0 . 6 5}$ & $\mathbf{0 . 9 2}$ \\
\hline 3 & BhartiAirtel & -2.03 & -1.22 & -1.10 & -0.79 & -1.57 & 2.08 & 1.75 & 1.94 & -2.44 & -1.63 & $\mathbf{- 0 . 4 0}$ & $\mathbf{1 . 5 5}$ \\
\hline 4 & BHEL & -4.27 & 0.74 & -1.07 & -1.72 & 1.37 & 1.65 & 3.06 & 1.18 & -0.39 & -2.70 & $\mathbf{- 0 . 0 8}$ & $\mathbf{1 . 9 9}$ \\
\hline 5 & Cipla & -0.67 & 0.31 & -0.07 & -0.41 & -2.00 & -2.05 & -0.43 & -0.55 & -1.82 & -1.41 & $\mathbf{- 0 . 5 2}$ & $\mathbf{0 . 9 9}$ \\
\hline 6 & Coal India & -0.90 & -1.62 & -0.74 & -1.38 & -0.05 & -3.81 & -1.15 & -1.20 & 1.83 & -1.32 & $\mathbf{- 0 . 3 0}$ & $\mathbf{1 . 6 1}$ \\
\hline 7 & DrReddy Lbs & 0.51 & -0.42 & 2.91 & -0.11 & -0.45 & 0.08 & 0.20 & -0.51 & -1.07 & -0.65 & $\mathbf{- 0 . 3 0}$ & $\mathbf{1 . 3 7}$ \\
\hline 8 & GAIL & -1.26 & -0.19 & -1.13 & -1.35 & -2.43 & -1.04 & 0.50 & 1.71 & -0.76 & -0.95 & $\mathbf{- 0 . 4 6}$ & $\mathbf{1 . 2 4}$ \\
\hline
\end{tabular}




\begin{tabular}{|c|c|c|c|c|c|c|c|c|c|c|c|c|c|}
\hline 9 & HDFC & -0.54 & -3.77 & -0.55 & -1.26 & -0.43 & 0.37 & 2.52 & 0.50 & -1.20 & -3.61 & $\begin{array}{l}-0.54 \\
\end{array}$ & 1.62 \\
\hline 10 & HDFC Bank & -0.40 & -1.87 & 0.15 & -1.22 & -1.83 & 1.22 & 4.15 & -1.29 & -1.55 & -1.85 & -0.41 & 1.57 \\
\hline 11 & Hero Motocorp & 0.17 & -0.80 & -1.90 & -0.75 & -1.85 & 2.37 & 0.46 & 0.10 & -2.09 & -0.88 & -0.24 & 1.24 \\
\hline 12 & Hindalco & 1.04 & -0.21 & -4.29 & $\begin{array}{l}-0.22 \\
\end{array}$ & 2.29 & 1.19 & 3.34 & 4.25 & -2.50 & -1.28 & 0.37 & 2.23 \\
\hline 13 & HUL & 0.09 & -0.86 & -1.47 & -0.28 & 1.45 & -0.45 & 2.56 & -0.58 & -1.69 & -1.06 & -0.42 & 1.17 \\
\hline 14 & ICICI Bank & -2.70 & -0.13 & -1.15 & -2.43 & 0.10 & 3.58 & 2.29 & 0.82 & -2.84 & -2.11 & -0.42 & 2.07 \\
\hline 15 & Infosys & 1.61 & -0.05 & 0.17 & $\begin{array}{l}-0.71 \\
\end{array}$ & -0.16 & 0.60 & 1.39 & 1.27 & -1.20 & -1.62 & 0.05 & 1.00 \\
\hline 16 & ITC & 0.47 & -0.30 & -0.80 & 0.87 & -1.66 & -0.02 & 3.61 & -0.72 & -0.76 & -2.59 & -0.41 & 1.66 \\
\hline 17 & Larsen & -0.68 & 1.84 & -3.08 & -1.57 & -1.03 & 2.20 & 3.90 & 1.82 & -1.77 & -2.86 & -0.13 & 1.96 \\
\hline 18 & M\&M & -0.31 & -1.86 & -0.94 & 0.97 & 1.83 & 3.36 & 2.07 & 0.11 & -0.44 & -0.88 & 0.28 & 1.80 \\
\hline 19 & Maruti Suzuki & -0.19 & -2.17 & 0.49 & -0.03 & -0.36 & 1.84 & 1.31 & 3.28 & -1.91 & -0.19 & 0.06 & 1.41 \\
\hline 20 & NTPC & -0.13 & 0.23 & -1.51 & -0.96 & 0.54 & 2.07 & 1.87 & -0.39 & -0.55 & -2.63 & 0.15 & 1.58 \\
\hline 21 & ONGC & 0.44 & -2.15 & -2.42 & -1.76 & 0.96 & 0.67 & 3.22 & -0.63 & -0.13 & -2.24 & -0.42 & 1.67 \\
\hline 22 & Reliance & -1.42 & -1.18 & -1.35 & -1.33 & -0.81 & 0.76 & 2.65 & 0.27 & -1.51 & -1.78 & -0.37 & 1.28 \\
\hline 23 & SBI & -3.17 & -0.46 & -2.25 & -1.73 & 1.34 & 1.45 & 2.60 & 3.18 & -1.52 & -1.97 & 0.18 & 2.64 \\
\hline 24 & Sesa Sterlite & 0.81 & 0.50 & -0.53 & -3.35 & -2.24 & 1.78 & -1.36 & -1.88 & 0.95 & -3.91 & -0.64 & 2.03 \\
\hline 25 & Sun Pharma & 0.47 & -0.63 & -0.86 & 0.21 & 1.61 & -1.34 & 1.73 & -0.66 & -1.42 & -2.60 & -0.40 & 1.50 \\
\hline 26 & Tata Motors & -2.76 & 1.29 & -2.04 & -4.53 & 1.58 & 5.52 & 1.65 & -0.40 & -1.28 & -0.83 & 0.08 & 2.37 \\
\hline 27 & Tata Power & -2.44 & 0.30 & -1.03 & -2.70 & -1.77 & 1.09 & 0.19 & 0 & 0.76 & -2.58 & -0.25 & 1.48 \\
\hline 28 & Tata steel & 3.64 & 2.86 & 1.25 & -2.15 & 1.86 & 4.58 & 2.86 & -0.74 & 0.48 & -0.62 & 1.08 & 1.95 \\
\hline 29 & TCS & 1.41 & -1.34 & 0.25 & -0.53 & -1.66 & -1.29 & 1.02 & -0.79 & -0.32 & -1.69 & -0.32 & 1.36 \\
\hline 30 & Wipro & 0 & -0.05 & -0.06 & 0.22 & -0.03 & 0.63 & 1.42 & -0.77 & -0.95 & -0.99 & 0.04 & 0.77 \\
\hline
\end{tabular}

As shown in the above table, before decrease in the interest rate, the average returns of the Infosys, M\&M, Maruti Suzuki, NTPC, Hindalco, SBI, Tata motors, Tata Steel and Wipro were positive. The average return of the Axis bank, Bajaj Auto, Bharthi Airtel, BHEL, Cipla, Cola India, DrReddy lab,
GAIL, HDFC, HDFC bank, Hero Motocorp, HUL, ICICI bank, ITC, ONGC, Reliance, Sesa Sterrlite, Sun Pharma, Tata Power, TCS, were negative. During this period standard deviation of the Axis Bank was the highest 2.72, and Wipro has lowest standard deviation of returns (0.77). Tata steel has the highest average returns.

Table 2: Average returns and standard deviation of returns, after decrease in the interest rate

\begin{tabular}{|l|l|l|l|l|l|l|l|l|l|l|l|l|l|}
\hline $\begin{array}{l}\text { SI } \\
\text { No. }\end{array}$ & COMPANY & $\begin{array}{l}\text { Nov- } \\
\mathbf{2 5}\end{array}$ & $\begin{array}{l}\text { Nov- } \\
\mathbf{2 6}\end{array}$ & $\begin{array}{l}\text { Nov- } \\
\mathbf{2 7}\end{array}$ & $\begin{array}{l}\text { Nov- } \\
\mathbf{2 8}\end{array}$ & $\begin{array}{l}\text { Nov- } \\
\mathbf{2 9}\end{array}$ & $\begin{array}{l}\text { Dec- } \\
\mathbf{0 2}\end{array}$ & $\begin{array}{l}\text { Dec- } \\
\mathbf{0 3}\end{array}$ & $\begin{array}{l}\text { Dec- } \\
\mathbf{0 4}\end{array}$ & $\begin{array}{l}\text { Dec- } \\
\mathbf{0 5}\end{array}$ & $\begin{array}{l}\text { Dec- } \\
\mathbf{0 6}\end{array}$ & AVG & STD \\
\hline 1 & Axis Bank & 2.97 & -0.13 & 0.46 & 0.08 & 3.06 & 3.09 & 0.11 & -0.22 & 4.33 & 3.18 & $\mathbf{1 . 6 9}$ & $\mathbf{1 . 7 7}$ \\
\hline 2 & Bajaj Auto & 2.37 & 0.07 & 0.39 & 0.19 & 1.22 & -0.50 & -0.47 & -1.14 & 0.33 & 0.55 & $\mathbf{0 . 3 0}$ & $\mathbf{0 . 9 7}$ \\
\hline 3 & Bharti Airtel & 0.1 & -2.72 & -1.76 & 0.57 & 1.10 & 1.56 & -0.50 & 0.48 & 0.96 & -0.98 & $\mathbf{- 0 . 1 2}$ & $\mathbf{1 . 3 6}$ \\
\hline 4 & BHEL & 5.22 & 2.57 & -0.98 & 3.04 & 3.45 & 2.05 & 2.57 & 0.46 & 3.93 & 0.47 & $\mathbf{2 . 2 8}$ & $\mathbf{1 . 8 5}$ \\
\hline 5 & Cipla & 0.65 & -0.35 & -1.00 & -0.55 & 2.92 & 0.13 & -0.28 & -0.12 & -0.65 & 0.23 & $\mathbf{0 . 1 0}$ & $\mathbf{1 . 1 0}$ \\
\hline 6 & Coal India & 0.75 & -2.45 & 0.84 & 1.70 & 0.65 & 0.72 & -1.02 & 0.39 & 2.87 & 3.58 & $\mathbf{0 . 8 0}$ & $\mathbf{1 . 7 3}$ \\
\hline 7 & DrReddysLabs & 0.15 & -1.31 & 0.77 & 1.27 & 1.15 & 0.56 & -1.39 & -0.56 & -1.52 & 0.36 & $\mathbf{- 0 . 0 5}$ & $\mathbf{1 . 0 7}$ \\
\hline 8 & GAIL & 1.20 & -1.08 & 0.08 & 0.88 & 1.91 & -1.42 & 2.97 & -1.05 & 1.31 & 0.61 & $\mathbf{0 . 5 4}$ & $\mathbf{1 . 4 2}$ \\
\hline 9 & HDFC & 3.14 & -1.53 & 0.14 & 0.81 & 1.23 & 0.31 & -0.50 & -1.22 & 1.79 & -1.65 & $\mathbf{0 . 2 5}$ & $\mathbf{1 . 5 5}$ \\
\hline 10 & HDFC Bank & 2.66 & -1.11 & 0.27 & -0.07 & 1.19 & -0.02 & -0.59 & 0.13 & 4.52 & -0.79 & $\mathbf{0 . 6 2}$ & $\mathbf{1 . 7 4}$ \\
\hline 11 & HeroMotocorp & 2.40 & 1.01 & 0.33 & 0.39 & -0.44 & 0.60 & -0.72 & -0.84 & 0.98 & 2.33 & $\mathbf{0 . 6 0}$ & $\mathbf{1 . 1 3}$ \\
\hline 12 & Hindalco & 0.13 & 0.04 & 0.21 & 2.36 & 1.03 & -0.69 & 1.64 & -2.50 & 0.95 & -1.07 & $\mathbf{0 . 2 1}$ & $\mathbf{1 . 4 0}$ \\
\hline 13 & HUL & 1.65 & 1.06 & -0.56 & 1.05 & 0.69 & -1.88 & -0.65 & -1.36 & -0.90 & -0.98 & $\mathbf{- 0 . 1 9}$ & $\mathbf{1 . 2 0}$ \\
\hline 14 & ICICI Bank & 5.07 & -2.61 & -0.29 & -0.24 & 2.71 & 1.97 & -0.26 & -1.93 & 6.66 & 0.59 & $\mathbf{1 . 1 7}$ & $\mathbf{2 . 9 6}$ \\
\hline
\end{tabular}




\begin{tabular}{|l|l|l|l|l|l|l|l|l|l|l|l|l|l|}
\hline 15 & Infosys & -0.65 & -0.26 & -0.70 & 0.86 & 0.89 & -0.45 & 0.30 & 0.49 & -0.41 & -0.73 & $\mathbf{- 0 . 0 7}$ & $\mathbf{0 . 6 4}$ \\
\hline 16 & ITC & 2.56 & -1.69 & 1.03 & -0.14 & 2.09 & 0.37 & -0.67 & -1.86 & -1.39 & 0.89 & $\mathbf{0 . 1 2}$ & $\mathbf{1 . 5 5}$ \\
\hline 17 & Larsen & 3.97 & -0.04 & 0.47 & 1.67 & 1.95 & 2.22 & -1.10 & -1.40 & 4.49 & 0.91 & $\mathbf{1 . 3 1}$ & $\mathbf{1 . 9 5}$ \\
\hline 18 & M\&M & 0.79 & 0.05 & -0.20 & 1.78 & -1.29 & 1.34 & -0.84 & -0.74 & 0.16 & 0.12 & $\mathbf{0 . 1 2}$ & $\mathbf{0 . 9 7}$ \\
\hline 19 & Maruti Suzuki & 2.43 & -1.00 & -0.31 & 0.31 & 0.87 & -0.96 & 0.14 & -1.35 & 3.67 & -0.20 & $\mathbf{0 . 3 6}$ & $\mathbf{1 . 5 9}$ \\
\hline 20 & NTPC & -0.46 & -0.77 & -1.41 & 0.78 & -0.30 & -0.03 & -1.09 & -0.17 & -0.96 & 3.44 & $\mathbf{- 0 . 1 0}$ & $\mathbf{1 . 3 9}$ \\
\hline 21 & ONGC & 3.70 & -0.57 & 0.98 & 0.88 & 2.17 & -1.54 & -0.58 & -1.66 & 1.20 & 1.31 & $\mathbf{0 . 5 9}$ & $\mathbf{1 . 6 9}$ \\
\hline 22 & Reliance & 0.88 & -1.09 & -0.49 & 1.23 & 0.57 & 0.21 & 0.82 & -0.65 & 1.58 & -0.30 & $\mathbf{0 . 2 8}$ & $\mathbf{0 . 8 8}$ \\
\hline 23 & SBI & 3.69 & -1.04 & -1.23 & -0.02 & 3.29 & 0.06 & -0.46 & 0.29 & 1.87 & 0.44 & $\mathbf{0 . 6 9}$ & $\mathbf{1 . 7 1}$ \\
\hline 24 & Sesa Sterlite & 0.17 & 1.00 & -1.22 & 0.57 & 4.42 & 0.44 & -1.36 & 0.28 & -0.52 & 0.86 & $\mathbf{0 . 4 6}$ & $\mathbf{1 . 6 1}$ \\
\hline 25 & Sun Pharma & 0.92 & -1.25 & -0.57 & 0.18 & 0.19 & 4.09 & -0.50 & -0.19 & -2.24 & 0.67 & $\mathbf{0 . 1 3}$ & $\mathbf{1 . 6 7}$ \\
\hline 26 & Tata Motors & 3.28 & 1.23 & 2.31 & -0.31 & 0.15 & 0.31 & -0.14 & -1.58 & -0.69 & 0.18 & $\mathbf{0 . 4 7}$ & $\mathbf{1 . 4 4}$ \\
\hline 27 & Tata Power & 1.62 & -0.70 & -0.19 & 0.71 & 1.79 & 0.57 & 0.13 & 2.44 & 1.77 & 5.57 & $\mathbf{1 . 3 7}$ & $\mathbf{1 . 7 8}$ \\
\hline 28 & Tata steel & 1.40 & -1.41 & 0.73 & 1.52 & 0.02 & 1.90 & 0.18 & 1.25 & 1.74 & 0.26 & $\mathbf{0 . 7 6}$ & $\mathbf{1 . 0 2}$ \\
\hline 29 & TCS & 0.60 & -0.86 & -0.52 & -0.02 & 1.01 & 0.48 & 0.30 & -0.87 & -0.62 & 0.49 & $\mathbf{- 0 . 0 0}$ & $\mathbf{0 . 6 8}$ \\
\hline 30 & Wipro & 0.20 & -0.82 & -1.06 & 0.63 & -0.40 & 2.24 & 0.15 & 1.95 & 0.18 & 0.17 & $\mathbf{0 . 3 2}$ & $\mathbf{1 . 0 7}$ \\
\hline
\end{tabular}

From the above table we can find that after decrease in the interest rate, the average returns of Axis Bank, Bajaj Auto, BHEL, Cipla, Cola India, GAIL, HDFC, HDFC bank, Hero Motocorp, Hindalco, ICICI Bank, ITC, Larsen, M\&M, Maruti Suzuki, ONGC, Reliance, SBI, Sesa Sterlite, Sun Pharma, Tata Motors, Tata Power, Tata steel and Wipro were positive. The average returns of the Bharti Airtel, Dr Reddy lab, HUL, Infosys, NTPC and TCS were negative. During this period standard deviation of the L\&T was the highest 2.72, and Infosys has the lowest.

\section{FINDINGS OF THE STUDY:}

It is found that most (21) of the stocks had negative returns before decrease in the interest rate. But after decrease in the interest rates, most of the stocks (24) have earned positive returns. The following stocks have positive returns: Axis Bank, Bajaj Auto,
BHEL, Cipla, Cola India, GAIL, HDFC, HDFC bank, Hero Motocorp, Hindalco, ICICI Bank, ITC, Larsen, M\&M, Maruti Suzuki, ONGC, Reliance, SBI, Sesa Sterlite, Sun Pharma, Tata Motors, Tata Power, Tata steel and Wipro are positive. The study shows the fact that most of the stocks have positive returns both before and after increase in the interest rates. It is also found that the returns after decrease in the interest rates will be higher than the returns during increase in the interest rates. Therefore it can be concluded that the investors are more motivated during decrease in the interest rates. The reason for this is that the investors are able to borrow at lower interest rates during decrease in the interest rates and the borrowed amount will be invested in the stock market with the expectation of higher return from the share market. Thus, share market reacts positively for decrease in the interest rates.

\section{BIBLIOGRAPHY:}


[1] Balla V. K. (2002), Portfolio Analysis and Management, Sulthan Chand and Co. Ltd., New Delhi.

[2] BhatSudhindra (2008), Security Analysis and Portfolio Management, Excel Books, New Delhi.

[3] Booie.Zvi, Kane Alex and et.al.(2006), Investments, $6^{\text {th }}$ edition, Tata McGraw Hill, New Delhi.

[4] Chandra Prasanna (2008), Investment Analysis and Portfolio Management, $3^{\text {rd }}$ edition, Tata McGraw Hill, New Dehi.

[5] Fisher. E. Donald and Jordan. J. Ronald (2006), Security Analysis and Portfolio Management, Pearson Prentice Hall.

[6] http://www.bseindia.com/markets/eq uity/EQReports/StockPrcHistori.aspx ?flag $=0$ \&expandable $=7$

[7] http://www.rbi.org.in/scripts/BS_Vie wBulletin.aspx ? $\mathrm{Id}=15232$

[8] Kevin. S (2008), Portfolio Management, $2^{\text {rd }}$ edition, PHI Learning Pvt. Ltd, New Delhi.

[9] PandianPunithavathy (2004), Security Analysis and Portfolio Management, Vikas Publishing House Pvt. Ltd, New Delhi. 